\title{
A NEW PROOF THAT TEICHMÜLLER SPACE IS A CELL
}

\author{
A. E. FISCHER AND A. J. TROMBA
}

\begin{abstract}
A new proof is given, using the energy of a harmonic map, that Teichmüller space is a cell.
\end{abstract}

In [2] the authors developed a new approach to Teichmüller's famous theorem on the dimension of the unramified moduli space for compact Riemann surfaces. Teichmüller's theorem states (roughly) that the space $T$ of conformally inequivalent Riemann surfaces of genus $p, p>1$ (with some topological restrictions) is homeomorphic to Euclidean $\mathbf{R}^{6 p-6}$. In proving homeomorphism Teichmüller had put a complete Finsler metric on this space. In [2] we showed that $\tau$ naturally carried the structure of a $C^{\infty}$ connected and simply connected differentiable manifold of dimension $6 p-6$. The proof of this was straightforward and used only splitting results for symmetric tensors and a standard existence theorem in elliptic partial differential equations. Using somewhat deeper results from the theory of harmonic functions between Riemannian manifolds and a result of Earle and Eells, we were then able to show that our moduli space $\tau$ was a contractible manifold.

The purpose of this note is to show that there is a straightforward proof that our Teichmüller space is diffeomorphic to $\mathbf{R}^{6 p-6}$. This completes the program of giving the main classical results of Teichmüller strictly in terms of concepts from Riemannian geometry as was formulated in $[\mathbf{2}, \mathbf{3}, \mathbf{4}]$.

\section{A quick review of the Fischer-Tromba approach to Teichmüller} theory. Let $M$ be a compact oriented surface without boundary. Let $C$ denote the space of complex structures compatible with the given orientation, $D$ the space of $C^{\infty}$ diffeomorphisms, $D_{0}$ those homotopic (and hence isotopic) to the identity, and $\mathcal{M}_{-1}$ those Riemannian metrics on $M$ with Riemann scalar curvature negative one. If $c=\left\{\varphi_{i}, U_{i}\right\}, \bigcup U_{i}=M$, is a complex coordinate atlas for $M$ and $f \in D$, then $\left\{\varphi_{i} \circ f, f^{-1}\left(U_{i}\right)\right\}$ is a complex coordinate atlas for $M$ which we designate as $f^{*} c$. If $g \in \mathcal{M}_{-1}$, then for each $x \in M, g(x): T_{x} M \times T_{x} M \rightarrow R$ is a positive definite symmetric quadratic form on $M$. By $f^{*} g$ we mean the form $g(f(x))(d f(x) \cdot, d f(x) \cdot)$.

One can then form the quotient spaces $\mathcal{M}_{-1} / D, \mathcal{M}_{-1} / D_{0}, C / D, C / D_{0}$. The main result of $[2]$ is

THEOREM 1.1. The spaces $T=\mathcal{M}_{-1} / D_{0}$ and $C / D_{0}$ naturally have the structure of a $C^{\infty}$ connected and simply connected finite-dimensional manifold of dimension

Received by the editors September 15, 1986.

1980 Mathematics Subject Classification (1985 Revision). Primary 32G15, 53C21; Secondary $32 \mathrm{C} 10,30 \mathrm{~F} 15$.

Key words and phrases. Teichmüller space, Dirichlet's functional, harmonic maps, holomorphic quadratic differentials, geometric methods. 
6p-6. Moreover there is a naturally defined equivariant diffeomorphism from $\mathcal{M}_{-1}$ to $C$ which passes to a diffeomorphism of $\mathcal{M}_{-1} / D_{0}$ with $C / D_{0}$. The space $C / D_{0} \cong$ $\mathcal{M}_{-1} / D_{0}$ is the Teichmüller space of $M$.

We should remark that the true Riemann space of moduli $R=M_{-1} / D=C / D$ is not a smooth manifold but does have the structure of an algebraic variety.

For purposes of exposition we wish to describe how one puts a differentiable structure on $\mathcal{M}_{-1} / D_{0}$ and to see what the natural tangent space is to this manifold. To see how the diffeomorphism between $\mathcal{M}_{-1} / D_{0}$ and $C / D_{0}$ is constructed the reader is referred to [3]. Let us think of $\mathcal{M}_{-1}$ as an infinite dimensional submanifold of the space of $C^{\infty}$ symmetric two tensors $S_{2}$ on $M$. For $g \in \mathcal{M}_{-1}$, the tangent space $T_{g} O_{g}$ to the orbit $O_{g}$ of $D_{0}$ at $g$ consists of all symmetric tensors of the form $L_{X} g$, the Lie derivative of $g$ with respect to some vector field $X$ on $M$. In this case $X$ will be uniquely determined by $h \in T_{g} \mathcal{M}_{-1}$. The next splitting result of symmetric tensors is basic to our theory.

THEOREM 1.2. Every $h \in T_{g} \mathcal{M}_{-1}$ can be expressed uniquely as a direct sum $h=h^{T T}+L_{X} g$ where $h^{T T}$ is a symmetric two tensor on $M$ which is trace free and divergence free. This implies that in a conformal coordinate system (with respect to the metric $g$ ), $h^{T T}$ has a local representation as

$$
h^{T T}=u d x^{2}-u d y^{2}-2 v d x d y=\operatorname{Re}\left\{(u+i v)(d x+i d y)^{2}\right\}
$$

where $u+i v$ is a holomorphic function of the local coordinates $z=x+i y$, and $\operatorname{Re}$ designates the real part.

Thus every $h \in T_{g} \mathcal{M}_{-1}$ can be expressed uniquely as a direct sum

$$
h=\operatorname{Re}\left(\xi(z) d z^{2}\right)+L_{X} g
$$

where $\xi(z) d z^{2}$ is a holomorphic quadratic differential on $M$ with respect to the complex structure induced by $g$. Moreover every such holomorphic quadratic differential occurs in decomposition (1).

Now the $C^{\infty}$ manifold structure on $\mathcal{M}_{-1} / D_{0}$ follows as a consequence of fact that $D_{0}$ acts freely and that as a consequence of the theorem of Riemann-Roch the space of holomorphic quadratic differentials on $M$ has finite dimension $6 p-6$.

We summarize these facts as

THEOREM 1.3. The tangent space to the manifold $\mathcal{M}_{-1} / D_{0}$ at an element $[g] \in$ $\mathcal{M}_{-1} / D_{0}$ can be naturally identified with those symmetric two tensors which are trace free and divergence free and also (by taking real parts) with the holomorphic quadratic differentials on $M$, holomorphic with respect to the complex structure induced by $g$.

As we already stated, we are viewing $\mathcal{M}_{-1}$ as a differentiable submanifold of the space of all symmetric tensors $S_{2}$. There is a natural "weak" $L_{2}$ Riemannian structure $\langle\langle\rangle$,$\rangle on \mathcal{M}_{-1},\langle\langle,\rangle\rangle_{g}: T_{g} \mathcal{M}_{-1} \times T_{g} \mathcal{M}_{-1} \rightarrow R$ defined by

$$
\left\langle\left\langle h^{1}, h^{2}\right\rangle\right\rangle_{g}=\int_{M} h^{1} \cdot h^{2} d \mu(g)
$$

where, $m$ local coordinates,

$$
h^{1} \cdot h^{2}=g^{a b} g^{c d} h_{a c}^{1} h_{b d}^{2}
$$


and where $\left\{g^{a b}\right\}$ denotes the local representation of the inverse to the matrix $\left\{g_{i j}\right\}$ of $g, d \mu(g)$ is the volume element of $g$, and where the Einstein summation convention is used. One can also give an intrinsic formulation of (2) avoiding local coordinates, as follows.

Using the metric $g$ we can transform $h^{1}, h^{2}$ into $1: 1$ tensors $H^{1}, H^{2}$ satisfying

$$
g(x)\left(H_{x}^{i} X_{x}, Y_{x}\right)=h^{i}(x)\left(X_{x}, Y_{x}\right), \quad i=1,2,
$$

for all $X_{x}, Y_{x} \in T_{x} M$. Then each $H^{i}$ is symmetric with respect to $g$ and for $x \in M$ the $\operatorname{trace} \operatorname{tr}\left(H_{x}^{1} H_{x}^{2}\right)$ is a well-defined function (of $\left.x\right)$ on $M$. Then (2) is equivalent to

$$
\left\langle\left\langle h^{1}, h^{2}\right\rangle\right\rangle_{g}=\int_{M} \operatorname{tr}\left(H^{1} H^{2}\right) d \mu(g) .
$$

This $L_{2}$-Riemannian metric is $D$ invariant, a fact which follows immediately from the change of variables formula. Thus $D$ acts on $\mathcal{M}_{-1}$ as a group of isometries.

The important remark is that (1) is an $L_{2}$-orthogonal decomposition.

2. Dirichlet's functional on Teichmüller space. Let $g_{0} \in \mathcal{M}_{-1}$ and $\left[g_{0}\right]$ denote its class in $\mathcal{M}_{-1} / D_{0}$. This fixed $g_{0}$ will act as our base point. Let $g \in \mathcal{M}_{-1}$ be any other metric and let $s: M \rightarrow M$ be viewed as a map from $(M, g)$ to $\left(M, g_{0}\right)$. Using the metrics $g$ and $g_{0}$ one defines Dirichlet's energy functional

$$
E_{g}(s)=\frac{1}{2} \int_{M}|d s|^{2} d \mu(g)
$$

where $|d s|^{2}=$ trace $_{g} d s \otimes d s$ depends on both metrics $g$ and $g_{0}$, and again $d \mu(g)$ is the volume element induced by $g$.

We may assume that $\left(M, g_{0}\right)$ is isometrically embedded in some Euclidean $\mathbf{R}^{k}$, which is possible by the Nash-Moser embedding theorem. Thus we can think of $s:(M, g) \rightarrow\left(M, g_{0}\right)$ as a map into $\mathbf{R}^{k}$ with Dirichlet's integral having the equivalent form

$$
E_{g}(s)=\frac{1}{2} \sum_{i=1}^{k} \int_{M} g(x)\left\langle\nabla_{g} s^{i}(x), \nabla_{g} s^{i}(x)\right\rangle d \mu(g) .
$$

For fixed $g$, the critical points of $E$ are then said to be harmonic maps. From $[1,5$ and 8] we have the following result.

THEOREM 2.1. Given metrics $g$ and $g_{0}$ there exists a unique harmonic map $s(g):(M, g) \rightarrow\left(M, g_{0}\right)$. Moreover $s(g)$ depends differentiably on $g$ in any $H^{r}$ topology, $r>2$, and $s(g)$ is a $C^{\infty}$ diffeomorphism.

Consider the function $g \rightarrow E_{g}(s(g))$. This function on $\mathcal{M}_{-1}$ is $D$-invariant and thus can be viewed as a function on Teichmüller space. To see this one must show that $E_{f *}\left(s\left(f^{*}(g)\right)\right)=E_{g}(s(g))$. Let $c(g)$ be the complex structure associated to $g$ given by Theorem 1.1. For $f \in D_{0}, f:\left(M, f^{*} c(g)\right) \rightarrow(M, c(g))$ is a holomorphic map, and consequently since the composition of harmonic maps and holomorphic maps is still harmonic, we may conclude, by uniqueness, that $S\left(f^{*} g\right)=S(g)$ 。 $f$. Since Dirichlet's functional is invariant under complex holomorphic changes of coordinates, it follows immediately that

$$
E_{f^{*}(g)}(s(g) \circ f)=E_{g}(s(g)) .
$$


Consequently for $[g] \in \mathcal{M}_{-1} / D_{0}$, define the $C^{\infty}$ smooth function $\tilde{E}: \mathcal{M}_{-1} / D_{0} \rightarrow R$ by $\tilde{E}([g])=E_{g}(s(g))$. We wish now to prove the main theorem of this note, namely

THEOREM 2.2. Teichmüller space $\mathcal{M}_{-1} / D_{0}$ is $C^{\infty}$ diffeomorphic to $\mathbf{R}^{6 p-6}$.

To prove this result it suffices to show that $\tilde{E}$ has the following properties:

(i) $\tilde{E}$ is a proper map, i.e. the inverse image of bounded sets in $\mathbf{R}$ under $\tilde{E}$ is compact in $\mathcal{M}_{-1} / D_{0}$.

(ii) $\left[g_{0}\right]$ is the only critical point of $\tilde{E}$.

(iii) $\left[g_{0}\right]$ is a nondegenerate minimum.

Once (i) through (iii) are established the result follows immediately from the application of the well-known gradient deformations of Morse theory.

The proof of (i) follows from ideas due to Mumford, Schoen and Yau [7], and a result on equicontinuity of harmonic maps (Jost [6, p. 20]). Using a result of Mumford, Schoen and Yau show that $E: \mathcal{M}_{-1} / D_{0} \rightarrow R$ is proper; that is, $E$ is proper on the true space $R$ of Riemann moduli.

Now suppose that $\tilde{E}\left[g_{n}\right]$ is a bounded sequence. It then follows from [7] that $\left\{g_{n}\right\}$ represents a sequence of a class of metrics in $\mathcal{M}_{-1} / D_{0}$ all of whose injectivity radii are strictly bounded below. By a version of Mumford's theorem due to TomiTromba [10], it follows that there is a subsequence of $g_{n}$, call it again $g_{n}$ and a sequence of diffeomorphisms $f_{n} \in D$ such that $f_{n}^{*} g_{n}$ converges.

Let $\gamma_{n}=f_{n}^{*} g_{n} ; r_{n}=s_{n} \circ f_{n}$. Then $E\left(\gamma_{n}, r_{n}\right)$ is a bounded sequence of real numbers, the $\gamma_{n}$ all have injectivity radii strictly bounded below, and $r_{n}:\left(M, \gamma_{n}\right) \rightarrow$ $\left(M, g_{0}\right)$ is harmonic. We claim that one can find a subsequence $f_{n}$ all of which are in the same homotopy class.

Suppose not. Then there is a subsequence of the $f_{n}$ all in distinct homotopy classes. Again call the subsequence $f_{n}$. From Jost's result, the $r_{n}=s_{n} \circ f_{n}$ are equicontinuous. Since the $s_{n}$ are all homotopic to the identity, this gives a contradiction.

Thus we may assume the $f_{n}$ are in one homotopy class, $f_{n}=h_{n} \circ f, f \in D$ fixed and $h_{n} \in D_{0}$. Then necessarily $h_{n}^{*} g_{n}$ (or more simply $\left[g_{n}\right]$ ) converges. This proves properness on $\mathcal{M}_{-1} / D_{0}$.

To show (ii), again let $s=s(g):\left(M, g_{0}\right) \rightarrow\left(M, g_{0}\right)$ be the unique harmonic map determined by $g$ and $g_{0}$. Let $\mathcal{N}_{g}(z) d z^{2}$ be the quadratic differential defined by

$$
\mathcal{N}_{g}(z) d z^{2}=\sum_{i=1}^{k} \frac{\partial s^{i}}{\partial z} \cdot \frac{\partial s^{i}}{\partial z} d z^{2}
$$

where $s^{i}$ is the $i$ th component function of $s:(M, g) \rightarrow\left(M, g_{0}\right) \hookrightarrow \mathbf{R}^{k}$, and $z=x+i y$ are local conformal coordinates on $(M, g)$. We next prove

THEOREM 2.3. $\mathcal{N}_{g}(z) d z^{2}$ is a holomorphic quadratic differential on $(M, c(g))$.

PROOF. Let $\Omega$ denote the second fundamental form of $\left(M, g_{0}\right) \subset \mathbf{R}^{k}$. Thus for each $p \in M, \Omega(p): T_{p} M \times T_{p} M \rightarrow T_{p} M^{\perp}$. Let $\Delta$ denote the Laplacian maps from $(M, g)$ to $\left(M, g_{0}\right)$, and $\Delta_{\beta}$ denote the Laplace-Beltrami operator on functions. Then if $s$ is harmonic we have

$$
0=\Delta s=\Delta_{\beta} s+\sum_{j=1}^{2} \Omega(s)\left(d s\left(e_{j}\right), d s\left(e_{j}\right)\right)
$$


with $e_{1}(p), e_{2}(p)$ an orthonormal basis for $T_{p} M$ (w.r.t. the matrix $g$ ). $\mathcal{N}_{g}$ will be holomorphic if

$$
\frac{\partial}{\partial \bar{z}}\left(\sum_{i=1}^{k} \frac{\partial s^{i}}{\partial z} \cdot \frac{\partial s^{i}}{\partial z}\right)=0
$$

But this equals

$$
2 \sum_{i=1}^{k} \Delta_{\beta} s^{i} \cdot \frac{\partial s^{i}}{\partial z}
$$

and by (4) we see that this in turn equals

$$
\begin{aligned}
& -2 \sum_{i=1}^{k} \sum_{j=1}^{2} \Omega^{i}(s)\left(d s\left(e_{j}\right), d s\left(e_{j}\right)\right) \cdot \frac{\partial s^{i}}{\partial z} \\
& \quad=-2 \sum_{i=1}^{k} \sum_{j=1}^{2}\left\{\sum \Omega^{i}(s)\left(d s\left(e_{j}\right), d s\left(e_{j}\right) \frac{\partial s^{i}}{\partial x}\right)+i \Omega(s)\left(d s\left(e_{j}\right), d s\left(e_{j}\right) \frac{\partial s^{i}}{\partial y}\right)\right\} .
\end{aligned}
$$

Since $\Omega(p)$ takes value in $T_{p} M^{\perp}$ it follows that both the real and the imaginary parts of this expression vanish.

From 1.2 we saw that $\xi=\operatorname{Re}\left(\mathcal{N}_{g}(z) d z^{2}\right)$ is a trace free divergence free symmetric two tensor on $(M, g)$. Let $\rho \in T_{[g]} \mathcal{M}_{-1} / D_{0}$. We know from 1.3 that we may think of $\rho$ as a trace free divergence free symmetric two tensor. From [10] we have the following result:

THEOREM 2.4. DE $([g]) \rho=-\langle\langle\xi, \rho\rangle\rangle_{g}$. Thus $[g]$ is a critical point of $\tilde{E}$ if $\rho=0 \equiv \operatorname{Re}\left(\mathcal{N}_{g}(z) d z^{2}\right)$, or if $\mathcal{N}_{g}(z) d z^{2} \equiv 0$.

THEOREM 2.5. $\mathcal{N}_{g}(z) d z^{2}=0$ implies that $[g]=\left[g_{0}\right]$.

PROOF. $\mathcal{N}_{g}(z) d z^{2}=\left\{\left|s_{x}\right|^{2}-\left|s_{y}\right|^{2}+2 i\left\langle s_{x}, s_{y}\right\rangle\right\} d z^{2}$. Thus $\mathcal{N}_{g}(z) d z^{2}$ implies that $s$ is weakly conformal. Since $s$ is a diffeomorphism it is conformal. Thus $s:(M, c(g)) \rightarrow\left(M, c\left(g_{0}\right)\right)$ is holomorphic and hence $[g]=\left[g_{0}\right]$.

It remains to show (iii). It is clear that since $\mathcal{N}_{g}(z) d z^{2} \equiv 0\left(s\left(g_{0}\right)=\right.$ id) that $\left[g_{0}\right]$ is a critical point.

Let $\rho, \nu \in T_{\left[g_{0}\right]} \mathcal{M}_{-1} / D_{0}$ be trace free, and divergence free symmetric two tensors. Then a straightforward computation yields

THEOREM 2.6. The second derivative or Hessian of $\tilde{E}$ at $\left[g_{0}\right]$ is given by the formula

$$
D^{2} \tilde{E}\left(\left[g_{0}\right]\right)(\rho, \nu)=2 \int_{M} \rho \cdot \nu d \mu\left(g_{0}\right)=2\langle\langle\rho, \nu\rangle\rangle_{g_{0}} .
$$

Thus the Hessian of $\tilde{E}$ at $\left[g_{0}\right]$ is essentially the natural inner product on $T_{\left[g_{0}\right]} \mathcal{M}_{-1} / D_{0}$ and hence a positive definite quadratic form. This concludes the proof of our main result 2.2 .

\section{REFERENCES}

1. J. Eells and L. Lemaire, Deformations of metrics and associated harmonic maps, Petodi Memorial Volume, and preprint IHES.

2. A. E. Fischer and A. J. Tromba, On a purely Riemannian proof of the structure and dimension of the unramified moduli space of a compact Riemann surface, Math. Ann 267 (1984), 311-345. 
3. __ Almost complex principal fibre bundles and the complex structure on Teichmüller space, J. Reine Angew. Math. 352 (1984), 151-160.

4. __ On the Weil Petersson metric on Teichmüller space, Trans. Amer Math. Soc. 284 (1984), 319-335.

5. P. Hartmann, On homotopic harmonic maps, Canad. J. Math. 19 (1967), 673-687.

6. J. Jost, Harmonic maps between surfaces, Lecture Notes in Math., vol. 1062, Springer-Verlag, Berlin and New York, 1984.

7. R. Schoen and S. T. Yau, Existence of incompressible minimal surfaces and the topology of three dimensional manifolds with non-negative scalar curvature, Ann. of Math. (2) 110 (1979), $127-142$.

8. __ On univalent harmonic maps between surfaces, Invent. Math. 44 (1978), 265-278.

9. M. Shiffman, The Plateau problem for minimal surfaces of arbitrary topological structure, Amer. J. Math. 61 (1939), 853-882.

10. F. Tomi and A. J. Tromba, A geometic proof of the Mumford compactness theorem, Proc. of the DDZ Symposium on Partial Differential Equations, Nankai Univ., 1986, Springer-Verlag (to appear).

Department of Mathematics, University of California at Santa Cruz, SANTA CRUZ, CALIFORNIA 95064

Max Planck Institut für Mathematik, Gottfried-Claren-Strasse 26, 5300 BONN 3, WEST GERMANY 